\title{
Bericht über das Helgoländer Arbeitstreffen der deutschen Gewässermikrobiologen, Juni 1964
}

\author{
Zusammenstellung unter Verwendung von Zusammenfassungen \\ der Teilnehmer
}

WILFRIED GUNKEL

Biologische Anstalt Helgoland, Meeresstation, Helgoland

\begin{abstract}
Report on the Helgoland working-group meeting of German aquatic microbiologists, June 1964 (prepared from summaries submitted by the participants). In order to stimulate scientific contacts between the few German microbiologists devoted to studying the ecology of aquatic microorganisms, and to work out agreements on methodical problems, a meeting was held at the marine station of the Biologische Anstalt Helgoland from June 24 to 26,1964 . The main topic discussed was: "The methods used in aquatic microbiology". The present report contains summaries of the papers presented, some of the discussions and a brief description of different microbiological methods demonstrated by the participants (different types of samplers, sampling from board of the research vessel "Uthörn", culture media, counting techniques and determination of bacterial activity). The second meeting on the same subject will be held in Plön, West Germany, in 1965.
\end{abstract}

\section{EINLEITUNG}

Die wissenschaftlichen Kontakte zwischen den wenigen in Deutschland auf dem Gebiet der ökologischen Gewässermikrobiologie arbeitenden Wissenschaftlern sind unzureichend. Zudem wird dieses Fachgebiet auf den üblichen Symposien und Kongressen meistens nur am Rande behandelt. Um hier Abhilfe zu schaffen, wurde von GuNKEL ein Arbeitstreffen der Gewässermikrobiologen vorgeschlagen. Dieser Vorschlag fand allgemeine Zustimmung. So kam es zu dem ersten Arbeitstreffen der deutschen Gewässermikrobiologen, welches in der Zeit vom 24. bis 26. Juni 1964 auf Helgoland in den Räumen der Meeresstation der Biologischen Anstalt Helgoland abgehalten wurde.

Das Arbeitstreffen sollte den kleinen Kreis der in Deutschland aktiv und unmittelbar auf dem Gebiet der ökologischen Gewässermikrobiologie arbeitenden Spezialisten zusammenführen. Das Thema der Arbeitstagung lautete: „D i e M e tho d e n in der Gewässermikrobiologie ". Vorträge, Diskussionen und zahlreiche Demonstrationen stimulierten einen fruchtbaren Gedanken- und Erfahrungsaustausch, über dessen wesentlichste Punkte hier kurz berichtet werden soll. 
Folgende Wissenschaftler nahmen an dem Arbeitstreffen teil (Abb. 1):

Dr. F. Gerring, Institut für Biochemie und Technologie der Bundesforschungsanstalt für Fischerei, Hamburg

Dr. M. GILlbricht, Biologische Anstalt Helgoland, Zentrale, Hamburg-Altona

Dr. W. Gunkel, Biologische Anstalt Helgoland, Meeresstation, Helgoland

Dr. E. Hagmeier, Biologische Anstalt Helgoland, Meeresstation, Helgoland

Dr. V. MEYER, Institut für Meeresforschung, Bremerhaven

Dr. J. Overbeck, Hydrobiologische Anstalt der Max-Planck-Gesellschaft, Plön

Dr. G. Rheinhelmer, Institut für Meereskunde der Universität Kiel, Kiel

Dr. R. Ruschкe, Limnologisches Institut der Universität Freiburg, Falkau im Breisgau

Dr. H. Scharfer, Biologische Anstalt Helgoland, Meeresstation, Helgoland

Dr. E. Thofern, Hygienisches Institut der Universität Göttingen, Göttingen

Dr. A. UlkEN, Institut für Meeresforschung, Bremerhaven

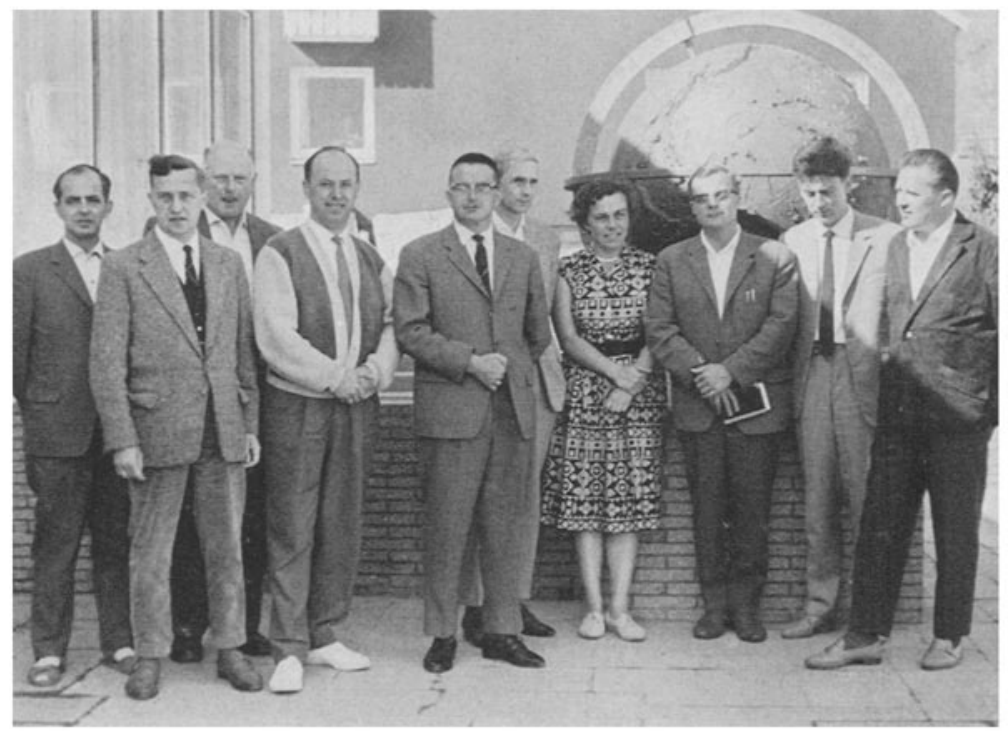

Abb. 1: Die Teilnehmer am Arbeitstreffen der deutschen Gewässermikrobiologen auf Helgoland. V. L. n. r.: Schaefer, Girlbricht, Meyer, Thofern, Gunkel, Rheinheimer, Ulken, Overbeck, Ruschke, Getrring; auf der Fotografie fehlt Hagmeier

\section{VERLAUF DES ARBEITSTREFFENS}

Nach einer zwanglosen Zusammenkunft der Teilnehmer am Vorabend begann der offizielle Teil des Arbeitstreffens am 24. Juni 1964 um 9.00 Uhr.

Einleitend sprach Herr GuNkel über die dringende Notwendigkeit eines verstärkten Erfahrungsaustausches über die auf dem Gebiet der ökologischen Gewässermikrobiologie angewandten Methoden. Die einzelnen Bearbeiter verwenden vielfach unterschiedliche Methoden, welche dann verschiedene, nicht unmittelbar vergleichbare 
Resultate liefern. Dieser unglückliche Umstand stellt ein erhebliches Handikap dar für die ökologische Gewässermikrobiologie, welche ja auf die vergleichende Betrachtung und Analyse von Ergebnissen aus verschiedenen Gegenden und Habitaten in besonderem Maße angewiesen ist. D i e beste Methode gibt es nicht. Jede Methode hat ihre Vor- und Nachteile, wobei im Gegensatz zur chemischen Analytik kaum Absolutwerte erwartet werden können, sondern eine starke Abhängigkeit von der jeweils angewandten Technik in Rechnung gestellt werden muß.

In den meisten Fällen hängt der Wert einer Untersuchung weit mehr von der Versuchsplanung $a b$ als von der im einzelnen gewählten Methode, sofern sie überhaupt brauchbar ist und in exakt der gleichen Weise standardisiert angewandt wird. Bei ökologischen Untersuchungen kommt es vor allem auf die Ermittlung von relativen Werten, und zwar möglichst zahlreichen an, wobei zwar eine hohe Genauigkeit der Einzelbestimmung sehr erwünscht, aber keineswegs immer erforderlich ist, da in vielen Fällen die Unterschiede der Standortfaktoren Zehnerpotenzen betragen und diese Werte häufig wechseln. Daher sind viele etwas gröbere Einzelbestimmungen in der Regel wertvoller als wenige sehr genaue, falls die Genauigkeit mit einem erhöhten Aufwand erkauft werden muß.

Das Ziel des Arbeitstreffens sollte sein, die Methoden anderer Bearbeiter genau kennenzulernen, um in der Lage zu sein, diese unter standardisierten Bedingungen parallel zu den eigenen anzuwenden, um so Vergleiche mit anderen Versuchsreihen, die mit dieser anderen Methode gewonnen wurden, anstellen zu können. Das Treffen sollte sich nicht auf rein mikrobiologische Arbeitsweisen beschränken, da auch andere Bestimmungen von uns durchgeführt werden müssen, etwa um unsere Werte in ökologische Kreisläufe eingliedern zu können. Diese anderen Bestimmungen sind insbesondere notwendig: erstens als Indikatoren, beispielsweise für bestimmte Wasserkörper oder Wasserströmungen, zweitens für das Erkennen der Wirksamkeit von Milieufaktoren und drittens zur Erfassung der Ausgangssubstanzen beziehungsweise Stoffwechselprodukte im Rahmen der Umsetzungsprozesse.

Das Arbeitstreffen sollte sich insbesondere befassen mit der Probenentnahme, der Aufarbeitung der Proben sowie mit Hilfsbestimmungen, Teilproblemen also, denen für das Endergebnis ganz besondere Bedeutung zukommt.

Anschließend berichtete Herr Gunker über das Arbeiten an Bord von Forschungsschiffen. Auf dem neu in Dienst gestellten Forschungsschiff "Meteor" ist ein bakteriologisches Laboratorium vorhanden, das ausgezeichnete Arbeitsbedingungen bietet. Es ist vollständig mit Kunststoffplatten verkleidet, besitzt Kühlschrank und Gefriertruhe, fließend warmes, kaltes und kochendes Süßwasser sowie Seewasser; zwei Schlingertische sind in die Arbeitstische eingebaut. Anschlüsse für Wechselstrom und stabilisierten Wechselstrom sind vorhanden. Im benachbarten Autoklavenraum befinden sich zwei Autoklaven, zwei Heißluftsterilisatoren (Memmert) sowie ein Ionenaustauscher. Zwei Wasserbäder und eine Haltevorrichtung für 2 Gasflaschen sind ebenfalls vorhanden. Zwei Brutschränke befinden sich im Kühlraum und erlauben, bei jeder gewünschten Temperatur zu bebrïten. UV-Lampen sorgen für Keimfreiheit. Ebenfalls sind auf den Fischereiforschungsschiffen "Anton Dohrn“ und „Walther Herwig“ bakteriologische beziehungsweise mikrobiologische Laboratorien eingerichtet. Es wäre sehr zu begrüßen, wenn zukünftig auch Kollegen aus dem Binnenlande Gebrauch von diesen 
auch im internationalen Bewertungsmaßstab ausgezeichneten Laboratorien machen würden, um die Möglichkeiten, die der bakteriologischen Forschung hiermit gegeben wurden, voll auszunutzen. Die Anzahl der reinen Meeresmikrobiologen ist zu gering, um ständig diese Labors zu besetzen. Da auf den Forschungsfahrten laufend die Milieufaktoren untersucht werden (z. B. Temperatur, Salzgehalt, Plankton) stehen diese Werte auch für die eigene Auswertung zur Verfügung.

Außerdem wies Herr Gunkel auf die ausgezeichneten Arbeitsbedingungen für Gastforscher an der Biologischen Anstalt Helgoland hin, die in noch stärkerem Maße von Mikrobiologen ausgenützt werden sollten. Eine größere Anzahl Laboratorien von der Deutschen Forschungsgemeinschaft in großzigiger Weise mit Apparaten und Glassachen ausgerüstet - steht für diesen Zweck das ganze Jahr über zur Verfügung. Die Schiffe der Biologischen Anstalt Helgoland sichern die Versorgung mit Untersuchungsmaterial. Bakterien der verschiedensten physiologischen Gruppen können leicht erhalten werden, und es bestehen Erfahrungen in der Isolation und Verbreitung, beispielsweise von Chitinabbauern, Agarverdauern, Sulfatreduzenten, Purpurbakterien, Schwefelwasserstoffoxydierern, Leuchtbakterien und Hefen.

Gleichfalls hingewiesen wurde auf die AQUATIC INTEREST GROUP innerhalb der American Society for Microbiology und dem von Professor S. Meyers, Miami, herausgegebenen "Newsletter". Es wurde vereinbart, daß Gunkel die Namen der Teilnehmer und deren Arbeitsgebiete der Gruppe zur Kenntnis bringt, um einen ständigen Kontakt herzustellen.

Anschließend wurde über Probenentnahme unter sterilen und unsterilen Bedingungen diskutiert. Für einwandfreie mikrobiologische Untersuchungen muß unbedingt die Verwendung eines sterilen Schöpfers gefordert werden. Die Nachteile bei der Zusammenarbeit mit anderen Disziplinen (Verwendung zusätzlicher anderer Schöpfer, zeitlich oder räumlich etwas abweichende Entnahme) müssen in Kauf genommen werden.

In letzter Zeit sind von SorokIN Untersuchungen mit sterilen Schöpfern gemacht worden in Gebieten, die bereits von Kriss unter Verwendung von Nansen-Schöpfern bearbeitet worden waren. Sorokrn kommt zu grundsätzlich verschiedenen Ergebnissen wie KRIss und ist der Ansicht, daß sämtliche Untersuchungen von KRISs und seinen Mitarbeitern angezweifelt werden müssen, da die Wasserproben durch den Schöpfer verunreinigt worden seien.

An der Biologischen Anstalt Helgoland wird seit sechs Jahren vom Vortragenden ein für sterile Entnahme geringfügig modifizierter Schöpfer nach ZoBELL verwandt, der in Serie eingesetzt wird. Er hat sich ausgezeichnet bewährt und arbeitet absolut zuverlässig. Als Aufnahmegefäße dienen dickwandige Bierflaschen, die bis $150 \mathrm{~m}$ Wassertiefe eingesetzt werden können. Ab dieser Tiefe werden Gummibälle als Aufnahmegefäße verwandt. Dem Schöpfer nach Zobell liegt das 1936 von ScHACH an der Biologischen Anstalt Helgoland entwickelte Prinzip des Zerschlagens eines durch einen Vakuumschlauch mit der Flasche verbundenen Glasrohres durch das Fallgewicht zugrunde.

Außerdem wurde der Schöpfer nach ISSATSCHENko besprochen sowie der von Niskin in Miami neuentwickelte bakteriologische Wasserschöpfer, der nach dem Blasebalgprinzip in der gewünschten Tiefe einen sterilisierten Plastikbeutel mit der Wasser- 
probe vollsaugt, demonstriert. Der Beutel wird nach Füllung durch ein Federwerk wieder verschlossen. Er gestattet die Entnahme von 41 Wasser.

Herr Overbeck demonstrierte ein von Sorokin für limno-bakteriologische Arbeiten als Endschöpfer entwickeltes Gerät, das nach einem ähnlichen Verfahren wie der ZOBELL-Schöpfer arbeitet.

Herr RHEINHeIMER führte ein von ihm bei seinen Elbuntersuchungen verwandtes Gerät vor, das nach dem Prinzip der Meyerschen-Schöpfflasche arbeitet.

Herr HagmeIER demonstrierte die schwermetallfreien Wasserschöpfer nach VAN DORN, den sogenannten „Meteor “-Schöpfer sowie die Metallgeräte nach NANSEN und Petterson, die bei hydrographischen Untersuchungen Anwendung finden. Außerdem erläuterte er die Arbeitsweise des geschützten und ungeschützten Kippthermometers.

Nachmittags sprach Herr RreinheImer zum Thema: Methoden zur mikrobiologischen Untersuchung von Flüssen. Es wurde über die Methoden berichtet, die bei einer mehrjährigen mikrobiologischen Untersuchung der Elbe in dem Abschnitt zwischen Schnackenburg und Cuxhaven Anwendung fanden. Zur Orientierung wurden zunächst bei zahlreichen Längsprofilfahrten Bestimmungen der Gesamtkeim-, Coli- und Hefezahl nach der Plattenmethode vorgenommen. Die Direktauszählung auf Membranfiltern war wegen des hohen Detritusgehalts im Flußwasser nicht möglich. Daher wurden Fotoprotokolle von Membranfilter-Präparaten charakteristischer Wasserproben hergestellt. Es zeigte sich, daß der Bakteriengehalt des Elbewassers starken jahreszeitlich bedingten Schwankungen unterliegt mit winterlichem Maximum und sommerlichem Minimum.

$\mathrm{Da}$ eine artenmäßige Analyse der in einem Gewässer vorhandenen Mikroorganismen noch nicht möglich ist, muß man sich im allgemeinen darauf beschränken, festzustellen, welche physiologischen Bakteriengruppen vorhanden sind und in welcher Menge sie sich in dem betreffenden Gewässer befinden. Altere Autoren (Minder 1918, KLEIN \& STEINER 1929 und andere) ermittelten die "Indirekte Aktivität", das heißt, sie gaben jeweils eine kleine Wasserprobe zu einer großen Menge Nährlösung und prüften dann nach meist längerer Bebrütungszeit auf bestimmte mikrobielle Umsetzungen. BAIER (1936) führte dann "Direkte Aktivitätsbestimmungen" durch, indem er eine kleine, konzentrierte Nährstoffmenge einer größeren Wasserprobe zusetzte und nach möglichst kurzer Bebrütungszeit die Prüfung vornahm.

Herr Rhernheimer modifizierte die BaIersche Methode und bezeichnete sie als Potenzbestimmung, da sie vor allem ein Ausdruck für die Zahl, nicht aber für die Aktivität der Mikroben im Gewässer ist. Dementsprechend ergibt sich im allgemeinen eine weitgehende Übereinstimmung der Potenzwerte und der durch Verdünnungsreihen ermittelten absoluten Zahlen. Unter extremen Bedingungen können allerdings Abweichungen vorkommen, so beispielweise bei anhaltend starker Kälte. Im allgemeinen kann man aber mit Hilfe solcher Potenzbestimmungen die Verteilung der wichtigsten physiologischen Bakteriengruppen in einem Gewässer recht gut verfolgen. Über die Aktivität dieser Bakteriengruppen im Gewässer selbst ist aber nur dann eine Aussage möglich, wenn die Potenzwerte mit den entsprechenden Daten der chemischen Wasseranalyse verglichen werden.

Bei seinen Elbeuntersuchungen ermittelte RHEInHEIMER regelmäßig folgende Potenzen: Ammonifikationspotenz (Fäulnisbakterien), Nitritationspotenz (Nitritbakte- 
rien), Nitratationspotenz (Nitratbakterien), Denitrifikationspotenz (Denitrifizierer), Sulfurikationspotenz (Thiobacillen).

Während es in den meisten Fällen notwendig ist, das Ausmaß der bakteriellen Umsetzungen in den Gewässern auf indirektem Wege festzustellen, kann beispielsweise der Zelluloseabbau dort direkt beobachtet werden, indem man die Zerreißfestigkeitsabnahme eines in das Wasser eingehängten Baumwollgarnes (Nm 15/50) prïft. Um mechanische Beschädigungen der Fäden zu verhindern, wurden diese bei den Elbeuntersuchungen in durchlöcherten Bleiröhren befestigt, die an Bojen oder eisernen Pontons befestigt werden konnten. Mit Hilfe der erwähnten Methoden war es möglich, einen Einblick in die wichtigsten bakteriellen Umsetzungen im Elbstrom zu gewinnen (RHerNHEIMER, Arch. Hydrobiol. [Suppl. Bd. 29 1964]).

Frau UlkeN bemerkte in der Disk us sion: Bei der Bestimmung der Keimzahlen von Nitrifikanten hat Herr RHEINHEIMER die Verdünnungsmethode in Verbindung mit der Aktivitätsbestimmung versucht. Dabei kommt er zu dem Schluß, daß offenbar ein relativ geringer Keimgehalt genügt, um wesentliche Umsetzungen zu bewerkstelligen. Nach meinen Untersuchungen muß hierbei berücksichtigt werden, daß diese Organismen in ziemlich starken Zusammenballungen leben. Das bedeutet, daß bei der letzten Verdünnung nicht ein Keim, sondern ein Klumpen von sehr vielen Bakterien erfaßt wird.

Herr Gunkel gab einige Beispiele zur Bestimmung der Bakterienzahlen verschiedener physiologischer Gruppen im marinen Miliea mit Hilfe von Verdünungsreihen, der sogenannten MPN-(most-probable-number-) Methode. Diese Methode ist gut geeignet, insbesondere bei der Bestimmung der Anzahlen von anaeroben Bakterien, zum Beispiel Sulfatreduzenten, da anaerobe Wuchsbedingungen leicht durch das vollständige Füllen kleiner, fest verschließbarer Schraubfläschchen mit sauerstofffreien Medien (unter Umständen nach Durchperlen von Wasserstoff) erreicht werden und eine sehr eindeutige Reaktion, zum Beispiel starke Trübung oder bei Sulfatreduzenten Schwarzfärbung des gesamten Flascheninhaltes durch Bildung von Eisensulfid ergeben. Anwendung fand diese Methode ebenfalls bei der quantitativen Bestimmung ölabbauender Meeresbakterien. Da kein Agar benutzt wird, ist die Gewähr gegeben, daß die einem rein anorganischen, flüssigen Nährmedium beigefügten Ole tatsächlich die alleinige Kohlenstoff- und Energiequelle für die Bakterien darstellen.

Als Vergleich zu den von RHEINHEIMER für die Aufnahme von Baumwollnetzgarnen zur Feststellung der Reißfestigkeitsabnahme verwandten Bleirohren wurde ein Kunststoffzylinder mit Grundgewicht demonstriert, der zum gleichen Zweck auf Helgoland benutzt wurde.

In einem Abendvortrag berichtete Herr Scraefer über: Vork o m m e n, B ed eutung und Isolierung der gelösten organischen Substanzen im Meerw asser. Bisher wurde eine größere Anzahl von organischen Verbindungen im Meerwasser qualitativ nachgewiesen, und wir nehmen heute an, daß fast das gesamte Spektrum der in marinen Organismen vorkommenden organischen Stoffe audh im Meerwasser vorhanden ist. Allerdings ist die Konzentration von vielen Stoffen so gering, daß sie mit den heute üblichen chemischen Methoden kaum nachweisbar sind. Über den Stoffumsatz im Meerwasser wissen wir noch sehr wenig, vermutlich spielen dabei die marinen Mikroorganismen eine große Rolle. Aus vielen Untersuchungen ist aber be- 
kannt, daß marine Organismen organische Verbindungen an das Wasser abgeben und auch aus dem Wasser aufnehmen. Die gelösten organischen Substanzen stellen wichtige Nahrungs- und Wachstumsfaktoren dar. Die Lebensweise mancher Meeresorganismen wird durch solche Spurenstoffe beeinflußt.

Uber die horizontale, vertikale und jahreszeitliche Verbreitung dieser Stoffe ist ebenfalls noch wenig bekannt, da hierzu quantitative Analysen durchgeführt werden müssen. Solche sind aber bisher nur in geringem Umfang durchgeführt worden. Einer der Gründe, warum wir heute noch so karge Kenntnisse über diese wichtigen Stoffe haben, ist in der Schwierigkeit ihrer Isolierung aus der Salzmasse zu suchen. Nur sehr wenige Substanzen können direkt nachgewiesen werden. Die meisten müssen erst isoliert und konzentriert werden, bevor man sie quantitativ nachweisen kann. Es sind bisher mehrere Methoden dazu vorgeschlagen und ausprobiert worden, jedoch ist die Höhe der Ausbeute häufig nicht quantitativ oder unbekannt. Daher wurde in der Meeresstation der Biologischen Anstalt Helgoland eine weitere Methode ausgearbeitet, die es gestattet, mit Hilfe des noch wenig bekannten Ionenverzögerungsverfahrens die Hauptmasse der Salze zu entfernen, wobei eine große Anzahl von im Modellversuch zugesetzten organischen Substanzen quantitativ wiedergefunden wird. Eine Restentsalzung kann beispielsweise durch Ionenaustauschmethoden erfolgen. Das neue Verfahren wurde eingehend beschrieben.

In Anschluß an den Vortrag von Herrn ScHaefer fand eine lebhafte D isk us sion statt, die sich vor allem mit der Bedeutung der gelösten organischen Stoffe in Meer- und Süßwasser und mit der Rolle der Mikroorganismen befaßte. Die Teilnehmer berichteten ferner über die Untersuchungen über gelöste organische Spurenstoffe in Gewässern, die in ihren Instituten durchgeführt werden.

Am Vormittag des 25. Juni fand bei strahlendem Sonnenschein und ruhiger See eine A u s f h r t statt mit dem Forschungskutter "Uthörn" der Biologischen Anstalt Helgoland, und zwar in das Gebiet der Tiefen Rinne, südwestlich von Helgoland. Hierbei wurde der größte Teil der am Vortage besprochenen Wasserschöpfer im Einsatz gezeigt, nämlich ZoBELL-Schöpfer in Serie, Blasebalg-Schöpfer nach Nrskrn, ein modifizierter Schöpfer nach COBET sowie die Schöpfer nach Sorokin, van DORN, NANSEN, PetTERson und der für das FS „Meteor" neu entwickelte Klappen-Schöpfer. Mit dem Stoßrohr nach Pratje wurde eine Sedimentprobe in ihrer natürlichen Schichtung entnommen,

Da sich die Bakterienpopulationen frisch entnommener Proben quantitativ und qualitativ in kurzer Zeit stark verändern, ist es notwendig, die Aufarbeitung sofort an Bord vorzunehmen. Aus diesem Grunde wurde von Herrn Gunkel die Anlage von Plattenkulturen und das mikrobiologische Arbeiten an Bord demonstriert, insbesondere wurde auf viele praktische Dinge hingewiesen, die erst ein einwandfreies Arbeiten unter sterilen Bedingungen an Bord erlauben, zum Beispiel auf den Schlingertisch, der es gestattet, auch bei stärkeren Rollbewegungen, die sich hauptsächlich bei gestopptem Schiff bei Arbeiten „auf Station“ unangenehm bemerkbar machen, zu arbeiten, sowie auf Grätings für Petrischalen, Pipetten und Entnahmeflaschen. Die Arbeitsweise eines schlingerunempfindlichen Autoklaven der Firma Webeke, der sich an Bord sehr bewährt hat und zur Verflüssigung des in Röhrchen abgefüllten Agars diente, sowie ein 
Wasserbad der Firma Rubarth, das auch bei Seegang einwandfrei regelt und zum Temperieren des Agars benötigt wird, wurden demonstriert.

Hingewiesen wurde ferner insbesondere auf die umfassende Versuchsvorbereitung, welche die Arbeit unter den erschwerten Bordbedingungen auf das unbedingt notwendige Maß beschränkt. Gezeigt wurden auch die Möglichkeiten, im Deckslabor Plattenverunreinigungen durch Desinfektionsmittel einzuschränken. Da seifenhaltige Mittel mit Seewasser starke Niederschläge bilden, wurde zur Desinfektion von Tisch, Wänden und Fußboden Quartamon (eine quarternäre Ammoniumverbindung) der Firma Schülke \& Mayer, das diesen Nachteil nicht aufweist, verwandt. Die Abtötung von Verunreinigung hervorrufenden Keimen in der Raumluft sowie an unzugänglichen Stellen (etwa Bulleiverschraubung, hinter Leitungen etc.) wurde durch Versprühen yon Luzol-Spray, ebenfalls von der Firma Schülke \& Mayer, erreicht.

Herr HAgmeier demonstrierte die Arbeitsweise des photoelektrischen Trübungsmessers nach JosEPH. Dieses Gerät besteht aus einer künstlichen Lichtquelle, deren Intensität nach Durchlaufen einer Meßstrecke im Wasser von einer Photozelle gemessen wird. Die wechselnde, die Photozelle erreichende Lichtmenge wird durch einen Schreiber an Bord registriert. Das Gerät gestattet eine kontinuierliche Aufzeichnung der Trübung der gesamten Wassersäule und damit auch geringfügige Inhomogenitäten beziehungsweise die genaue Position von Sprungschichten, die mit der punktförmigen Entnahme von Schöpfern nicht erkannt werden können.

Die Nachmittagssitzung eröffnete Herr Ruschke mit einem Vortrag über: B akteriologische Untersuchungen am Uberlinger See (Bodensee) von Herbst 1960 bis Frühjahr 1962. Gegenstand der bakteriologischen Untersuchungen war das Wasser, das vom Seepumpwerk Süßenmühle aus $60 \mathrm{~m}$ Tiefe gefördert wird. Zum Vergleich wurden auch das pelagiale Oberflächenwasser und der Filterschlamm geprüf. Es sollte hierbei der Gehalt an Fäkalindikatoren und die Keimzahl festgestell werden; daneben sollte untersucht werden, woher die Fäkalkeime in das Wasser aus $60 \mathrm{~m}$ gelangen. Insgesamt wurden 12 Probenentnahmen durchgeführt.

Die Keimzahlbestimmung erfolgte nach dem Plattenverfahren mit gemixten Wasserproben. Als Keimzahlen ergaben sich im Durchschnitt folgende Werte:

\begin{tabular}{cccc} 
& Agar $37^{\circ} \mathrm{C}$ & Agar $22^{\circ} \mathrm{C}$ & Gelatine \\
\hline Oberfläche & 16 & 104 & 117 \\
$60 \mathrm{~m}$ Tiefe & 11 & 82 & 51
\end{tabular}

Der Keimgehalt betrug in der Tiefe von $60 \mathrm{~m}$ rund $60 \%$ desjenigen der pelagialen Oberfläche. Die Anzahl der mesophilen Keime in der Tiefe betrug rund 70\% von jener des Oberflächenwassers. Dieser relativ geringe Unterschied in der Anzahl der bei $37^{\circ} \mathrm{C}$ wachsenden Keime zwischen Oberflächenwasser und Tiefenwasser ist bedeutsam, da man annehmen kann, daß ein Großteil dieser Keime aus Warmblüterfaeces stammt.

An Fäkalcoli wurden im Wasser aus $60 \mathrm{~m}$ Tiefe durchschnittlich $35 / 1$ gefunden. Das Minimum lag bei $2 / 1$ im Juli 1961, das Maximum bei 194/1 im März 1961. Der Durchschnittswert des Oberflächenwassers war rund $25 \mathrm{mal}$ höher, er betrug im Durchschnitt 174 Fäkalcoli/l. Als Fäkalcoli bezeichneten wir Coli I und Irregularis I, deren Reaktionen in der Bunten Reihe hier angeführt seien: 
Dextr. Lakt. Indol Methylr. VPR Citrat Bulier $44^{\circ} \mathrm{C}$

\begin{tabular}{llllllll}
\hline Coli I & 0 & 0 & + & + & - & - & 0 \\
Irreg. I & 0 & 0 & + & + & - & - & -
\end{tabular}

Der Enterokokkenbestimmung zur Beurteilung einer fäkalen Kontamination eines Gewässers wird ähnliche Bedeutung zugemessen wie dem Colinachweis. Wir fanden im Wasser aus $60 \mathrm{~m}$ Tiefe nur etwas weniger Enterokokken als im Wasser von der Oberfläche, nämlich 30/1 gegenüber 35/1. Der Durchschnittsgehalt an Enterokokken des Oberflächenwassers entspricht etwa $20 \%$, jener des Tiefenwassers etwa $87 \%$ des entsprechenden Fäkalcoligehaltes. Bei allen Proben zeigte sich jedoch, daß bei Zu- oder Abnahme des Fäkalcoligehaltes der Enterokokkengehalt ebenfalls erhöht oder verringert war.

Clostridium perfringens, das versport mit menschlichen und tierischen Fäkalien ins Wasser gelangt, wurde weder im Tiefen- noch im Oberflächenwasser häufig gefunden. Oft gelang der Nachweis nicht in Mengen bis zu 5 Litern. Als Höchstwerte fanden wir im Liter Oberflächenwasser 24 und im Liter Tiefenwasser 18 dieser Keime. P. aeruginosa stellt in den letzten Jahrzehnten einen immer häufigeren Befund im klinischbakteriologischen Untersuchungsmaterial dar, parallel dazu geht die Häufigkeit von Funden im Oberflächen- und Brunnenwasser. Ursache hierfür dürfte die weitgehende Antibiotikaresistenz dieser Keime sein. Es wird angegeben, daß 11 bis $15 \%$ der Bevölkerung Träger von $P$. aeruginosa sind. Mit den Exkrementen gelangen diese Keime ins Wasser. Im Durchschnitt wurden 2 bis $3 P$. aeruginosa im Liter Tiefenwasser gefunden. Im Wasser von der Oberfläche waren es durchschnittlich 21/1. Als Höchstwerte konnten im Tiefenwasser 12 dieser Keime im Liter nachgewiesen werden, im Oberflächenwasser 156/1. Micrococcus pyogenes aureus konnte trotz Untersuchung von jeweils bis zu 10 Litern Wasser nur einmal gefunden werden, nämlich in 51 Tiefenwasser.

Im Filterschlamm konnten alle Keime nachgewiesen werden, welche auch aus Wasser aus $60 \mathrm{~m}$ Tiefe isoliert wurden. Der Keimgehalt betrug 10000 bis $25000 / \mathrm{mg}$ Schlammtrockensubstanz. Bei den Fäkalcoli waren es 1 bis 22 pro $\mathrm{mg}$ Trodkensubstanz, bei den Enterokokken durchschnittlich 1/mg Trockensubstanz. P. aeruginosa fand sich gegenïber Coli und Enterokokken relativ häufig, nämlich bis zu $4 / \mathrm{mg}$ Trodkensubstanz. Eventuell tritt im Filterschlamm eine Vermehrung dieser Keime ein, wahrscheinlich überleben sie jedoch besser als Coli und Enterokokken. Auch C. perfringens war häufig zu finden, 2 bis $6 \mathrm{Keime} / \mathrm{mg}$ Trodkensubstanz. Es erwies sich damit erwartungsgemäß als allen übrigen untersuchten Keimen überlegen in seiner Uberlebensfähigkeit im Filterschlamm.

In der Nähe des Seepumpwerkes Süßenmühle wurden hauptsächlich im Winter in Massen auftretende Bläßhühner beobachtet; 21 daraufhin untersuchte Tiere enthielten pro Gramm Darminhalt 7000 bis 200000 Fäkalcoli. Der Enterokokkengehalt lag mit durchschnittlich $330000 / \mathrm{g}$ Darminhalt noch höher. C. perfringens wurden bis zu $11000 / g$ Darminhalt nachgewiesen. Bei einem der Tiere wurden auch pyogene Staphylokokken gefunden. Niemals dagegen wurde $P$. aeruginosa im Darminhalt der Bläßhühner nachgewiesen.

Die Hauptinfektionsquelle stellen jedoch die Abwässer dar, die von Uberlingen aus mit einer ufernahen Strömung in Richtung des nur etwa $5 \mathrm{~km}$ entfernten Seepump- 
werkes verfrachtet werden. Dieser "Abwasserstrom" dürfte sich, seinem spezifischen Gewicht zufolge, bis ins Hypolimnion einschichten. Welche Rolle als Keimtransporteur das Zooplankton spielt, ist noch nicht klar, es hat jedoch den Anschein als würde sich auf absterbendem Zooplankton in kürzester Frist eine spezielle Population proteolytischer Keime, hauptsächlich Pseudomonaden, ausbilden. Absterbendes Phytoplankton zeigte oft optisch einen starken Bakterienbesatz (Arbeiten von SonNABEND, Hyg.-bakt. Inst., Erlangen), wobei bislang nicht feststeht, ob es sich um Fäkalkeime handeln kann, oder ob es autochthone Wasserkeime sind.

Fluorescenten sind bekannt als typische Boden- und Wasserkeime. Verschiedene Arten bilden einen prostostatisch-protociden Wirkstoff. Wir untersuchten 298 Fluorescentenstämme aus dem Uberlinger See und aus Filterschlamm auf ihre besonderen Eigenschaften. Von diesen 298 Stämmen verflüssigten 174 Gelatine, alle bis auf 6 waren lecithinase-positiv. Bei den 124 Nichtverflüssigern, davon 97 P. putida, fanden sich nur 2 lecithinspaltende Stämme. Hemmstoffbildung gegenüber einer Amöbe vom LimaxTyp und Hämolyse wurde nur bei Gelatineverflüssigern beobachtet. Von den $174 \mathrm{Ge}-$ latineverflüssigern, unter denen sich $171 \mathrm{P}$. fluorescens fanden, erwiesen sich 115 als Hemmstoffproduzenten, das sind $68 \%$. Diese Untersuchungen erscheinen als nicht unwichtig zur Beurteilung der Vorgänge in biologischen Kläranlagen und zum Verständnis mangelhafter Bodenfiltration.

Herr Overbeck sprach uber das Thema: Zur Bakteriologie der Binnenseen, Methode und Ergebnisse. Es wurde eingangs darauf hingewiesen, daß unsere Kenntnisse der Bakterien im Meer- und Süßwasser immer noch verhältnismäßig gering sind und wir noch nicht einmal eine sichere Vorstellung von ihrer tatsächlichen Zahl haben. Die Ursache hierfür ist zweifellos in methodischen Schwierigkeiten zu suchen, vor allem darin, daß keine allgemein gïltigen Verfahren zur quantitativen Erfassung der Bakterien bestehen.

Die wichtigsten Methoden zur Bestimmung der Bakterienzahl sind 1. das KocHsche Plattenverfahren und 2. das Membranfilterverfahren. Im Vortrag wurde die Methodik der Membranfiltertechnik eingehend und kritisch erläutert und mit der Platten-

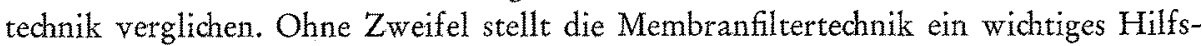
mittel zur Erfassung der Gesamtbakterienzahl dar, wenn auch nicht verkannt werden soll, daß das Ansprechen der gefärbten Bakterien auf dem durchsichtig gemachten Filter häufig schwierig ist und bei hohem Detritusgehalt des Gewässers unmöglich wird.

Das Plattenverfahren erfaßt grundsätzlich nur die saprophytischen Bakterien und ist zur Bestimmung der Gesamtbakterienzahl nicht geeignet.

Es wurden 2 Jahreszyklen von Bakterien und Phytoplankton, die mit Membranfiltern gewonnen wurden, vorgeführt und dabei gezeigt, daß anscheinend ein enger Zusammenhang zwischen Bakterien und Phytoplankton besteht. Denn in nicht verunreinigten Gewässern folgt die Zahl der Bakterien im wesentlichen den Schwankungen des Phytoplanktons: Bei ausgeprägten Frühjahrs- und Herbstmaxima des Phytoplanktons finden wir auch ebensolche der Bakterien. Im Winter ist die Zahl der Gewässerbakterien am geringsten. Im Hochsommer sind häufig Depressionen der Bakterienzahl festzustellen. In Obereinstimmung mit den mit direkter Bestimmung gewonnenen Bakterienzahlen anderer Autoren kann die Gesamtzahl der in einem Süßwasser vorhandenen. Bakterien mit rund 50000 bis $2000000 / \mathrm{ml}$ angenommen werden. 
Das Verhältnis der auf Keimplatten bestimmten Bakterien zu den auf Membranfiltern gezählten Bakterien schwankt stark in Abhängigkeit vom Reinheitsgrad des betreffenden Gewässers und kann maximal etwa $1: 30000$ betragen, das heißt einem Bakterium auf der Keimplatte entsprechen 30000 Bakterien auf dem Membranfilter. Je stärker verschmutzt ein Gewässer ist, desto geringer wird die Differenz zwischen den auf Keimplatten und den auf Membranfiltern bestimmten Bakterien. Der Vortragende gab seiner Überzeugung Ausdruck, daß in zukünttigen Gewässeruntersuchungen die Membranfiltertechnik in Kombination mit dem Keimplattenverfahren und der Bestimmung verschiedener physiologischer Aktivitäten unbedingt angewandt werden sollte.

An diesen Vortrag schloß sich eine äußerst rege $\mathrm{D}$ is $\mathrm{k} \mathrm{us} \mathrm{s} \mathrm{i} \mathrm{o} \mathrm{n} \mathrm{an,} \mathrm{die} \mathrm{nicht}$ in allen Einzelheiten festgehalten werden konnte. Unter anderem äußerte sich Herr MEYER, der folgendes ausführte: Die gewaltigen Unterschiede in den ermittelten Keimzahlen aus Plattenverfahren und Direktauszählung sind wahrscheinlich dadurch bedingt, daß für die Organismen der nicht verschmutzten Gewässer nicht geeignete Nährböden verwendet wurden. Es handelt sich offenbar vorwiegend um autotrophe Organismen, die mit der Plattenmethode kaum erfaßt werden. Hinzu kommt das langsame Wachstum dieser Bakterien. Man ist froh, wenn es gelegentlich gelingt, einen dieser Organismen überhaupt zu isolieren. Da die in der "Vegetationsschicht" zusätzlich erscheinenden Organismen wohl ebenfalls mit der Plattenmethode nicht erfaßt wurden, trat hier auch keine Erhöhung ein. Bei der direkten Methode wurden in der oberen Schicht 500000 Keime, in der "Vegetationszone" 900000 Keime und in der darunterliegenden Schicht 1200000 Keime gezählt. Das sind normalerweise keine signifikanten Unterschiede, denn man rechnet bei den Keimzählungen im allgemeinen nur nach Zehnerpotenzen. Eine andere Möglichkeit wäre die, daß die 500000 Keime der allgemeinen Population entsprechen, der sich in der Vegetationszone eine neue Artengruppe zugesellt hat, die sich darin dann aber sehr stark entwickelte. Da weiter mit der Direktmethode keine Unterbrechung $z$ wischen lebenden und toten Zellen gemacht werden kann, wäre in dieser Zone die Entwicklung der Bakterien im Vergleich zur Oberschicht unter Umständen noch stärker gewesen. Die noch höhere Keimzahl in der unteren Schicht ist vielleicht auf einen erhöhten Anteil toter Organismen zurüikzuführen, der aus den beiden anderen Schichten abgesunken ist. Wenn nach den Untersuchungen von Herrn Rheinheimer in stark belasteten Flüssen das Verhältnis von Plattenmethode zur Direktauszählung 1:1 ist gegenüber 1:1000 in den Seen, so liegt das offensichtlich daran, daß die Flüsse mit ihrer hohen Belastung an organischer Substanz (Eiweiß und Kohlenhydrate) vorwiegend mit solchen Organismen besetzt sind, die auf den üblichen Nährböden sehr gut gedeihen. Als Schlußfolgerung könnte gesagt werden, daß die Anwendung der Plattenmethode zur Voraussetzung hat, daß für die zu suchenden Organismen auch ein o p t i m a l e $\mathrm{r}$ Nährboden vorhanden sein muß. Bei autotrophen Organismen, die offensidhtlich in den Seen (auch in den sogenannten eutrophen Seen, die im Vergleich mit den meisten Flïssen als ausgesprochen sauber erscheinen) vorherrschen, gibt daher bis zur Auffindung spezifischer Nährböden nur die Aktivitätsbestimmung, wie sie beispielsweise Herr RHEINHeImer übt, den bestmöglichen Aufschluß über die Bakterienpopulationen.

Herr Gunkel äußerte sich zum Vortrag Overneck: $Z_{\text {war }}$ ist es sehr bedauerlich, daß mit der Gußplattenmethode nicht sämtliche Bakterien erfaßt werden, das ist 
jedoch ein Nachteil, von dem auch andere Richtungen, wie beispielsweise die Bodenbakteriologie und die Nahrungsmittelbakteriologie, betroffen werden, und diese Tatsache ist seit Jahrzehnten bekannt. Trotzdem werden allgemein die Kulturverfahren vorgezogen und auch die Kenntnisse, die wir uber die Verbreitung von Bakterien haben, beruhen fast ausschließlich auf diesen. Gegen die Direktzählmethode spricht meiner Ansicht nach folgendes:

1. Viele Bakterien im natürlichen Milieu haben nur sehr geringe Größe, die teilweise an der Grenze der Sichtbarkeit liegt. Hinzu kommt, daß zumindest im marinen Milieu Kokken und Kurzstäbchen, die nicht zu Gebilden wie Ketten, Tafeln etc. zusammentreten, was ihre Erkennung erleichtern würde, überwiegen. Gleichzeitig liegen häufig unbelebte Partikel gleicher Größenabmessung und Form vor, so daß eine klare Erkennung unmöglich ist.

2. Bei der großen Anzahl fraglicher Partikel werden verschiedene Bearbeiter unterschiedliche Ansichten darüber vertreten "was noch ein Bakterium ist"; es kommt ein subjektiver Faktor hinzu, der selbst mit der gleichen Methode gewonnene Ergebnisse verschiedener Bearbeiter oder Hilfskräfte nicht miteinander vergleichen läßt.

3. Lebende und tote Bakterien können nicht voneinander unterschieden werden, wenn auch gelegentlich behauptet worden ist, daß dies etwa durch Fluoreszensmikroskopie möglich sei.

4. Ganz unmöglich ist es, Bakterien zu erkennen und zu zählen, die sich auf oder unter Detritushaufen befinden, oder festzustellen, aus welcher Anzahl von Zellen ein Bakteriumaggregat besteht. Nun ist aber bekannt, daß in dem nährstoffarmen Milieu Meerwasser die Bakterien zum größten Teil gerade auf Partikeln, die im Wasser schweben, vorkommen, da nur hier eine die notwendige Schwellenkonzentration überschreitende Nährstoffmenge durch Oberflächenkräfte angereichert ist. Diese würden also nicht mit erfaßt.

5. Selbtsverständlich wachsen auf "normalen" Agarplatten beispielsweise keine Sulfatreduzenten und Purpurbakterien. Der Ausdruck "Gesamtbakterienzahl “ für die mit dieser Methode erhaltenen Werte ist also im Grunde genommen irreführend. Allerdings weiß jeder Mikrobiologe, was gemeint ist, wenn man sagt „die Gesamtbakterienzahl wurde mit Hilfe der Gußplattenmethode bestimmt". Viele Zahlen in der Bakteriologie sind ja sowieso nur in bezug auf die betreffende Methode zu verstehen. Eigentlich stimmt aber die Bezeichnung "Gesamtzahl" auch nicht für die Direktzählmethode, denn auch hier werden ja die Bakterien in Detritushaufen nicht mit erfaßt. Aber vielleicht könnte man die mit der Gußplattenmethode erfaßten Bakterien als aerobe Heterotrophe bezeichnen.

6. Bei Untersuchungen von Nährstoffkreisläufen dürften gerade die mit der Plattenmethode erfaßbaren Bakterien besonders interessant sein; denn gerade sie sind für einen Abbau von Eiweißkörpern und damit die Freisetzung etwa von N- und P-Verbindungen verantwortlich.

7. Der Zeitaufwand für die Durchführung einer Direktauszählung ist größer als für eine Keimzahlbestimmung mit Gußplatten. Insbesondere dürfte jedoch eine mikroskopische Durchmusterung von Membranfiltern wertvoll sein, um festzustellen, in welcher Form Bakterien vorliegen. - GuNKEL stimmte jedoch zu, daß es sehr sinnvoll wäre zu versuchen, beide Methoden bei denselben Proben anzuwenden, um Vergleiche an- 
stellen zu können. Eine gemeinsame Bearbeitung Helgoländer Meerwasserproben durch Gunkel und Overbeck wurde vereinbart. Abends hielt Herr A. Holtmann einen Lichtbildervortrag über das alte und neue Helgoland.

Herr Hagmeier berichtete über Die quantitative Bestimmung von Seston und dessen Eiweißgehalt. Beim feineren Filtrieren von Meerwasserproben gewinnt man nicht nur das Mikroplankton, sondern auch Tripton, Teilchen organischer und mineralischer Herkunft. Ein Maß für den Planktongehalt des Sestons gibt die Biuret-Reaktion, die photometrisch gemessen und mit Albumin geeicht werden kann. Die erhaltenen Albumin-Aquivalente lassen sich in Plankton-Trockengewicht und -Lebengewicht umrechnen. Filtrationen durch Papierfilter von etwa $1 \mu$ Porenweite ergaben in der Elbmündung bis zu $15 \mathrm{mg}$ Seston/Liter, bei Helgoland zwischen 1 und $5 \mathrm{mg}$, in der offenen Nordsee und im Nordatlantik um $1 \mathrm{mg}$, im tropischen Atlantik um 0,1 mg. Während in höheren Breiten und in Landnähe das Seston gewöhnlich zum größten Teil unbelebt ist, besteht es im offenen tropischen Ozean vornehmlich aus Plankton. Auf Papierfiltern gewonnene Sestonproben eignen sich außer zur Untersuchung auf den Eiweißgehalt zu Analysen von Pigmenten, Phosphor und der Menge organischer Substanz (Glïhverlust). Für Kohlenstoff-Bestimmungen benutzt man Filter aus Glasfasern. Mit dem Utermöhl-Mikroskop kann man aus dem in Kammern sedimentierten Seston Plankter und Partikel nach Zahl und Größe erfassen. Damit gewinnt man eine Vorstellung über die den Bakterien zur Siedlung verfügbaren Flächen. Die Beziehung des Sestongewichtes zur Extinktion des Meerwassers ist abhängig von der Größe der schwebenden Teilchen.

Im Anschluß an diesen Vortrag sprach Herr Gerring über Neue Methoden zur Bestimmung einiger physiologischer Gruppen von Bakterien. Für den Nachweis und für die Isolierung pektinspaltender Bakterien wird die Anwendung eines aus der Literatur bekannten, selektiv wirkenden Pektinmediums auch in der Gewässermikrobiologie vorgeschlagen und die Herstellungsweise eines solchen Selektivmediums mit dem Pektinpräparat "Rotbandpektin" bekanntgegeben. Pektinspaltende Bakterien bilden auf solchen Spezialnährböden makroskopisch erkennbare Verflüssigungszonen aus. In bezug auf Pektinzersetzer bietet die Anwendung der beschriebenen Methode die Möglichkeit, sowohl qualitativ als auch quantitativ zu arbeiten. Für den Nachweis des Zelluloseabbaues in Gewässern könnte möglicherweise das Präparat Tylose (= Carboxymethylzellulose) nützlich sein; entsprechende Versuche wurden angeregt. Zelluloseabbauende Mikroorganismen verändern den Viskositätszustand einer etwa 2\%igen Tyloselösung in kurzer Zeit in charakteristischer Weise. Für orientierende Versuche über den Zelluloseabbau in Gewässern ist der Einsatz dieses Präparates denkbar. Ohne viel Aufwand an Material und Arbeit könnte mit Hilfe dieses Präparates der Nachweis von Zellulosezersetzern in relativ sehr kurzer Zeit erbracht werden. Des weiteren wurde eine Arbeit referiert, die sich mit dem Nachweis der Gasbildung bei Bakterien nach einer vereinfachten Kapillar-Methode befaßt. Die Vorteile der genannten Kapillarmethode liegen darin, daß für die Untersuchungen nur kleinste Mengen Zucker benötigt werden, die Gärkapillaren nur geringen Raum beanspruchen und auch sehr kleine Gasmengen noch sicher erkannt werden.

Für die Identifizierung von Pseudomonaden wurde dieDurchführung der OxidaseReaktion erläutert und demonstriert. Die Oxidase-positive Reaktion ist innerhalb we- 
niger Sekunden ablesbar und für den Nachweis von Pseudomonaden charakteristisch. Zur serologischen Bestimmung von Bakterienarten wurde anhand eines Beispieles der Objektträger-Agglutinationstest mit einem entsprechenden Antiserum gezeigt und die Anwendung der serologischen Nachweismethode bei Bakterien empfohlen. Bei der Feststellung verwandtschaftlicher Beziehungen zwischen verschiedenen Mikroorganismenstämmen spielen unter anderem serologische Reaktionen eine wichtige Rolle.

Herr GILlbRicht hielt einen Vortrag über Die Chemie des Seew as sers und Rezepte zur künstlichen Herstellung von Seewasser für Versuchs$z$ wecke und Aquarien. Die verschiedenen veröffentlichten Seewasserrezepte unterscheiden sich im wesentlichen durch verschiedene Ionenkombinationen, Zusatz ausgesprodiener Nährsalze wie $\mathrm{NO}_{3}, \mathrm{HPO}_{4}$ etc., in bezug auf Spurenelemente und Komplexbildner. Mit p. a. Substanzen hergestelltes künstliches Seewasser enthält einen vielfach höheren Gehalt an Kupfer, das schädigend auf empfindliche Formen einwirkt. Bei Versuchen sollte weitgehend natürliches Seewasser Verwendung finden. In vielen Fällen dürften Salze technischer Reinheit den p. a. Substanzen vorzuziehen sein.

Herr Overbeck demonstrierte die Methoden zur Anfertigung von Präparaten für die Direktzählmethode von Bakterien (Filtration einer Seewasserprobe durch Membranfilter, Trocknung, Färbung mit Methylenblau, Durchsichtigmachen des Filters mit Immersionsöl, Mikroskopieren).

Die Teilnehmer berichteten über die zur Zeit in ihren Instituten laufenden Untersuchungen. Wie vorgeschlagen, tauschten sie vervielfältigte Listen der Veröffentlichungen, der Methoden sowie Nährböden- und Literaturzusammenstellungen aus.

Zum Abschluß dankte Herr Gunkel den Teilnehmern für die Vorträge, Diskussionsbeiträge und Demonstrationen und faßte die Ergebnisse kurz zusammen. Das Arbeitstreffen war ein Erfolg. Es hat zu einem besseren gegenseitigen Kennenlernen der Methoden und Arbeiten der Gewässermikrobiologen geführt und viele Anregungen gegeben. Von den Teilnehmern wurde der Wunsch ausgesprochen, diese Kontakte beizubehalten und das Treffen im nächsten Jahr zu wiederholen. Für 1965 lud Herr Overbeck nach Plön ein. 